\title{
Re-evaluating confidentiality: using patient information in teaching and publications ${ }^{\dagger}$
}

\author{
Heather Draper \& Wendy Rogers
}

\begin{abstract}
Case studies used in psychiatry for teaching and learning can include large amounts of personal information, and most medical journals refuse to publish case material without patient consent. However, not all patients are capable of consenting and maintaining anonymity is not always successful. Disclosure of personal information without consent can cause a sense of violation, but a principle of non-violation rigorously applied would prohibit the use of such material without consent, even if the patient is completely unidentifiable. A public interest argument can be made for using patient information for teaching purposes. Furthermore, a limited case can be made for using, and even publishing, some cases without consent, e.g. those requiring urgent public discussion or concerning people who are incapable of giving consent. Thus, an argument can be put forward for relaxing to this limited degree the demand for patient consent to publish case material.
\end{abstract}

One of us has been contributing for some years now to regular (about four times a year) sessions in an academic psychiatry department in which a real case is presented by a psychiatrist, and its ethical and legal implications are discussed with a lawyer. Every effort is made to anonymise these cases, although sometimes they are already in the public domain, for example owing to court proceedings or General Medical Council (GMC) hearings. The use of real cases seems to be a vital component in the success of these sessions (truth is often stranger and is certainly taken more seriously than fiction) and is typical in the teaching of psychiatry.

The use of case studies has several advantages. Potentially dry theoretical issues can be portrayed vividly, good practice disseminated, new or emerging issues highlighted and current practice challenged. Rarely, if ever, is the patient's consent sought. Is this a serious breach of confidentiality?

\section{Confidentiality: traditional views}

A justification for doctor-patient confidentiality can be found in most of the theories used in medical ethics.

${ }^{\dagger}$ For invited commentaries on this article see pp. 122-123 and pp. 123-124, this issue.

\section{Kantian ethics}

Kant reminds us of the absolute value of keeping our promises. In terms of medicine, this can be understood as an implied (Hippocratic) promise made by the medical profession that its members will not 'publish abroad' any personal details disclosed by any patient during the course of professional contact. Many professionals, including those who work with children, now point out to patients the limitations of this promise. Healthcare professionals have, for instance, statutory obligations to disclose matters such as child abuse. In effect, doctors should no longer promise that everything disclosed will be kept secret.

\section{Virtue ethics}

Virtue ethics reminds us of the importance of discretion in the development of our moral characters and habits. Here, discretion can be considered to be the golden mean between the two (habitual) vices of indiscretion and secretiveness. This analysis suggests that, on occasions, it could be wrong to adhere rigidly to confidentiality; for example, it might be necessary to involve close relatives in the care of a patient and impossible to do this without keeping them informed to a certain degree. On the

Heather Draper is Senior Lecturer in Biomedical Ethics at the University of Birmingham (Centre for Biomedical Ethics, Department of Primary Care and General Practice, Primary Care (T) Building, University of Birmingham, Edgbaston, Birmingham B15 2TT, UK. E-mail: h.draper@bham.ac.uk). She has been teaching medical/healthcare ethics (and some law) to medical students and postgraduates from a variety of healthcare backgrounds since the mid-1980s. She writes on a range of ethical issues, but her primary research focus is reproductive technology. Wendy Rogers is Associate Professor of Medical Ethics and Health Law in the School of Medicine at Flinders University, Adelaide, Australia. She currently teaches health law and ethics to students and practitioners in a range of healthcare professions. Her research interests include ethics of evidence-based medicine, ethical issues in general practice, feminist healthcare ethics and public health ethics. 
other hand, it is important only to disclose a minimum of information, only as much as is necessary to discharge one's obligations in caring for the patient or to meet legal obligations.

\section{Human rights}

The incorporation of the European Declaration of Rights into UK law has increased our understanding of rights theory and the significance of human rights. The Declaration reminds us that individuals have a right to privacy (a right to respect for private and family life, under Article 8), and that individuals who have privileged access to private information have a duty to ensure privacy, unless this right is outweighed by some competing right, such as the right to life. Thus, if a doctor knows that her patient poses a direct and immediate threat to the life of an identifiable person, that person's right to life may outweigh the patient's right to privacy. However, this disclosure must still respect the patient's privacy as far as possible and the doctor may be required not to disclose the patient's identity unless this is unavoidable to protect the life in danger (Stone, 2001).

\section{Consequentialism}

Consequentialism reminds us that we need to take account of the consequences of our actions, and that it is difficult to adhere rigidly to any moral rule. Maintaining confidentiality is vital to the operation of effective healthcare delivery. In psychiatry the importance of the patient's history - which may include the most personal of details - is obvious. Full and frank disclosure can be vital to diagnosis and treatment, but is unlikely to be forthcoming unless patients can trust doctors not to disclose this information indiscriminately. However, occasionally a balance has to be struck between the public interest in maintaining confidentiality and the public interest in disclosure, for example to prevent serious harm to an identified other. Notwithstanding the introduction of human rights legislation, English law generally reflects consequential thinking in emphasising the importance of confidentiality in the context of harms that may occur if confidentiality is privileged above other goods.

\section{Confidentiality in practice}

The general rule, then, is that patient confidentiality should be granted extreme respect, save when serious harm would follow, but it is also accepted that confidential information can be passed between
Box 1 Problems with current approaches to the use of patient information for publication or teaching

- Anonymisation (e.g. by de-identification) is not secure and this should be made clear to patients (see BMJ Publishing Group, 2004; Royal College of Psychiatrists, 2004)

- No one has the legal or ethical authority to consent to publication for incompetent adults (under English law)

- Authority of consent of individuals with parental responsibility for minors is unclear (no obvious best interests argument)

- It is not always clear to whom information belongs and, therefore, who should consent

- Use of patient information in unpublished teaching materials may breach confidentiality, but there are no official requirements for obtaining consent

healthcare professionals when this is necessary for the patient's care. Patients, however, can give permission for personal information to be disclosed more broadly than this.

Where does this leave us in terms of using patient information for teaching (Box 1)? The GMC recognises that it may be used for teaching purposes and does not seem to require that consent is sought (see 'Publishing abroad', below). Often, clinical teaching is intra-disciplinary, for example psychiatric teaching given by psychiatrists to psychiatric registrars or medical students. In these cases, the assumption seems to be that information can be shared between members of the same profession, who abide by the same standards in terms of respecting confidentiality. Multidisciplinary teaching can lead to sharing information with professionals who may not be bound by these same standards. Involving an ethics specialist, for instance, often entails including someone who is not a member of the medical profession. Ethicists of all people should recognise the importance of confidentiality, but they have no professional code governing their behaviour, nor a professional body ensuring that a professional code could be enforced.

Unlike sharing information for clinical care, teaching is only rarely in the immediate interests of the patient whose information is used (for instance, if the participants are involved in the patient's continuing care). Perhaps the breach of confidentiality could be justified on the basis of the greater good in the way that some research is justified. But if this is the case, why not obtain consent, or at least 
review by an independent body such as an ethics committee, as is required when private information is used for research? In the next sections, we discuss some of the challenges associated with obtaining consent to use patient information or anonymising the information to protect confidentiality.

\section{Obstacles to gaining consent}

Rogers \& Draper (2003) reviewed case studies in the Journal of Medical Ethics (JME) from 1982 to 2002, to determine whether or not authors mentioned that consent had been obtained from patients. The JME, unlike Advances in Psychiatric Treatment (Box 2), had no editorial policy on the publication of case studies.

The survey proved very instructive regarding the difficulties in obtaining consent. Of the 31 case histories found, in only just over half was it obvious that consent could have been obtained. In about a third of cases, the patients would not have been competent to consent. Some cases involved patients whose death formed part of the case history, making it impossible to obtain consent. In others, many people were involved and it was difficult to see whose consent was most relevant. For example, is it necessary to gain the consent of other healthcare professionals involved in the care of a patient when their attitudes, actions or behaviours are pertinent to the case? Finally, in some cases the issue under discussion concerned the propriety of disclosing certain information to patients. Therefore to have asked the patient's permission to discuss the case would have pre-empted the outcome of the discussion by disclosing the controversial information under debate.

Box 2 Summary of $A P T^{\prime}$ s instructions to authors regarding case studies

- Fictional cases are acceptable and even preferable

- For real cases, the patient's consent must be obtained, and proof of consent must be submitted

- The patient should review details before they are submitted

- If the patient is not able to consent, authorised proxy consent can be substituted

- Where there is no authorised proxy, the case must be anonymised such that no one (including a relative or carer) can identify the patient

- Simply altering age or treatment centre is not sufficient to protect confidentiality

\section{Patient refusal}

In psychiatry, there are particular reasons why patients might not consent to having their information used for teaching or publication. Mental illness continues to attract prejudice such that many patients may wish to keep this part of their life as private as possible. Reviewing details of disordered thoughts or psychotic episodes may serve as an unwelcome reminder for the patient. Raising the question of this kind of use of patient information may lead some patients to lose their trust in their confidentiality being maintained.

\section{Competence}

Given the episodic nature of much psychiatric illness, any discussion of a case should wait until the patient has recovered sufficiently to be deemed competent to consider consenting to this use of their information.

\section{Minors and patients lacking capacity}

Consent in the case of minors poses problems similar to those encountered with incompetent psychiatric patients, so it is worth considering the two groups together.

There are two moral and legal imperatives operating. First, parents in the UK have the legal privilege of consenting on behalf of incompetent minors to treatment, participation in clinical trials and use of case histories (but in England and Wales there is no legal proxy in the case of incompetent adults; Scotland has an Act for incompetent adults that permits some proxy consent). Second, clinicians are charged with acting in the interests of their incompetent patients, be they minors or adults.

If an intervention can reasonably be delayed until a minor is old enough, or sufficiently competent, to consent for himself or herself it is best practice to wait until this time. A similar delay would be best practice in the case of incompetent adults who are likely to regain capacity to consent.

Should this principle of delay be applied to the use of case histories in teaching and learning? The discussion of aspects of the care of an incompetent patient for teaching purposes is not in the interests of the patient concerned (although it might be in the interests of future patients). However, the value of publication or open discussion of a case in a teaching forum might be greatly reduced if it were delayed until a minor reached 18 years of age or an incompetent adult regained capacity. Long delays are likely to make a case significantly out of date. 
If their case is anonymised (see next section) it is arguable that no harm will come to the minor or incompetent adult involved, but it is not clear whether this is sufficient justification for discussing it without their (future) consent. Given that discussion of the case is not in the patient's medical interests, even though it is not contrary to these interests, it is not clear whether either the treating clinicians or the parents (in the case of children) are authorised to act on their behalf (although outside the medical context, parental consent - for instance to the publication of school photographs - would be the norm).

Thus if the rule on confidentiality were rigidly adhered to with children and incompetent adults, it would be unethical to discuss any case material, except in the context of the patient's medical care. This suggests that any discussion, however generally useful, of aspects of a minor's or incompetent adult's care should not be used in teaching or published. Clearly, however, paediatric cases are used both for teaching purposes and in publications (in the latter instance, usually with parental consent, even though it is not clear whether parents do actually have the ethical authority to consent). In the case of incompetent adults, because there is no legal proxy in England and Wales, journals have to choose between refusing without exception to publish without consent, or to allowing some exceptions but putting safeguards in place to ensure good practice. However, this cannot include some form of substituted consent, as this is legally meaningless as the English law stands.

There are also cases where conflicts of interests may arise. For instance, the actions or behaviours of other parties often form part of a psychiatric medical history. Sometimes these are reported by the patient, sometimes they are observed by the staff caring for the patient. In some cases, the patient's reports might be untrue or biased (consciously or unconsciously). Discussion of the patient's veracity or the extent to which the patient is interpreting actions and behaviours might form part of the case discussion. The patient might be happy for the case to be discussed, but those whose actions or behaviours are reported might not be similarly content. The alleged behaviours might show them in a bad light or they might be unhappy about the dissemination of what they perceive to be misinformation about them. They might feel that, although the information is part of the patient's medical record and the reports are clearly described as the patient's feelings or interpretations, they should nevertheless be entitled to refuse consent to information allegedly about them being disclosed outside the treating team. It is not clear, however, whether this is their information at all, and therefore to what extent they are entitled to review it or give consent to its use in teaching or research when the rules of confidentiality are applied.

\section{Obstacles to anonymisation}

One way of avoiding some confidentiality issues and protecting privacy is to anonymise the case. In psychiatry, however, this is no simple matter. Psychiatric case histories are of necessity very detailed. Even basic information such as gender or ethnic origin is likely to be significant and relevant. Removing the contextual details in order to ensure effective anonymisation is likely to reduce significantly the value of the case as a teaching or research tool. But the more detailed the information given, the more likely it is that the patient will be able to recognise herself or, perhaps more significantly, that others will be able to recognise her. Some authors state which details have been changed in order to protect patient anonymity, but ironically this can itself aid identification. Recognition is further helped by geographical clues provided by the author's name and affiliation.

Given the value of publications to individual and institutional prestige, it is probably unreasonable to expect authors to write anonymously. A requirement to do so in the interests of patient confidentiality would act as a disincentive to authors to write up cases for the benefit of the profession and future patients. In addition, the most interesting or valuable cases are likely to be unique, making anonymisation virtually impossible. Some patients may be able and willing to consent to publication, notwithstanding the limitations of anonymisation. Such consent would make publication permissible. But if the patient is unable to give consent, should the case history remain private between the patient and treating clinician? If a wider discussion of the case or dissemination of the care details would be in the interest of other patients, it may be in the public interest to consider using anonymised material without consent.

\section{Violation of privacy: the experience $v$. the principle}

Anonymisation has been the traditional method used to protect the patient's identity, even where the patient's consent has been obtained. This suggests that one of the central harms of disclosure is the experience of violation of privacy that comes with public exposure. Therefore anonymisation may counter this harm, even where consent has not been sought. 
For instance, assuming that the experience of violation is central to the harm of disclosure, what if a case seems to be my case but, because it has been anonymised, I can't be sure that it is my case? If I can't be certain that this case refers to me, to what extent has my privacy been violated? I could write and ask the authors whether they have used my details without my consent, but I might be one of six such patients to make the same enquiry. Even if the authors actually had my case in mind when writing the article, the fact that five other people thought that it referred to them suggests that anonymisation was successful. It is not obvious that, under these circumstances, I could justifiably complain. Consequently, it is not clear whether the authors should have obtained my consent when the anonymised case might refer equally well to other individuals.

Ineffective anonymisation can lead to the experience of violation. An urgency to create a rule about publishing patient information was generated when a psychiatric patient recognised herself in an article published in a medical journal (Court, 1995). The assumption here must be that if the patient recognised herself, others might recognise her too. For others to be certain of her identity, however, they would already have to be aware of certain facts about her to associate the 'case' with her; therefore at least some of her details would already be known to the reader. But this does not mean that the reader would not learn from the published case things that they did not know.

It is, however, worth noting that if the patient alone recognised herself and she chose not to mention this to anyone, no one else would learn any previously private information about her (as opposed to the 'case' they were reading about). In this respect, her sense of violation might be misplaced to the extent that she as an identifiable person is not exposed if no one apart from her knows that the case is about her. To counter this point it must either be accepted that it is the patient's sense of violation that counts, regardless of how justified this is, or that the experience or sense of violation is secondary to a principle of violation. The latter is discussed shortly; the former might fit these facts nicely, but could prove to be a hard rule to generalise to other circumstances. In addition, it raises the problem that patients might mistakenly 'recognise' themselves in a case and feel violated, even though they are not the person on whom the case was based.

A possible alternative to using real case studies is to generate credible fictional accounts. To do this, however, authors must have certain objectives in mind and if these are related to a case in their experience - one that raises particular clinical or ethical challenges, for instance - it is difficult to see how the case can be truly fictional. It is more likely that it will contain ideas both from the originating case and from others known at first or second hand. It will therefore be a composite case based in fact. If my case contributes to such a composite case, my information enters the public domain, even if no one including myself - associates it with me. To the extent that the information is not identifiable I am protected from a sense of violation of privacy, but nevertheless my information has been 'published abroad'.

There is a further danger in accepting that it is the experience of violation that is the significant harm, for this would suggest that if a patient were dead or so cognitively impaired as never to be able to have a sense of violation, there is no need to respect confidentiality. The thought that the confidentiality of patients who have died or are incompetent is due the same respect as that of competent, living patients suggests that it is the principle of violation that is significant, rather than its experience. If, however, it is really a matter of principle, then the confidentiality rule is very rigid indeed. Even epidemiological studies that rely on non-identifiable, anonymised data gained without consent would be violations despite the unlikelihood that any of the information could ever be traced back to an individual either by that individual or by others. The same would be true of composite case studies. Yet it is widely accepted that, for various reasons, anonymised data can and should be used in research without consent. For example, seeking consent may lead to bias in the population studied, leading to invalid results; and for large population studies, the cost of trying to contact all those involved may be prohibitive.

\section{'Publishing abroad': public interest on balance}

The GMC offers no explanation for adhering to its traditional position of permitting teaching using patient's personal information without consent. Its guidelines relating to the confidentiality of information about patients suggest:

'You should tell patients how information about them may be used to protect public health, to undertake research and audit, to teach or train clinical staff and students and to plan and organise healthcare services' (General Medical Council, 2004).

Further guidance is offered in relation to audit, but nothing more is said about teaching, although the GMC's general principle that unidentifiable information should be used wherever possible should probably be interpreted to cover teaching too. The specific objection of patients to use of their material for teaching purposes is not discussed, nor is any distinction between face-to-face teaching and publication of material. 


\section{Academic journals}

It is not obvious that it is always an unethical breach of confidentiality to publish case material in a medical or research journal without consent, particularly where it is unlikely that the patient or anyone else could make a positive identification. Indeed, it is arguable that the public interest in dissemination to the medical profession might outweigh even a patient's objection if the case poses unique issues. Although publication of a case in a medical journal does introduce that material into the public domain, we might consider this to be significantly different from publication in, for example, a tabloid newspaper. Nevertheless, academic journals can be accessed by the general public and they are regularly scanned by the media for 'newsworthy' stories.

\section{Teaching materials}

It might be argued that patient information used in face-to-face medical teaching and training remains within the domain of the same or related professions. But the distinction between using patient information in publications and in teaching might not be as robust as first appears: teaching materials can also enter the public domain. For instance, a case history used for training in one group may be carried by members of that group into other groups. Participants at a teaching session or conference may be more careless about the disposal or securing of training notes than their own patients' notes. Training packs and course handouts might easily, and innocently, be read by a student's flatmates. Overheads for meetings and lectures are sometimes placed on the internet. Even medical professionals might not take a disseminated case study to be as confidential as their own patients' records. The wider this dissemination, the more the patient's information is 'published abroad'. Bearing in mind the obstacles to anonymisation discussed above, it is likely that some patients will be identifiable even where efforts have been taken to avoid this.

These points obviously indicate that great care should be taken of training materials that include case studies. However, it is unlikely that they amount to a strong reason for not using case materials in teaching. The value of teaching, and the extent to which this value is enhanced by using actual cases, is likely to be regarded as outweighing the harm of accidental violations that may occur.

\section{What is in the public interest?}

One way to resolve the dilemma surrounding the use of patient information in medical education and research is to take a lead from guidance on the
Box 3 Summary of principles for recruitment in research trials of patients who lack capacity to consent

- The potential/actual harms must be minimal

- Competent patients could not be substituted

- Where possible, trials on competent patients should precede those on incompetent patients

- The research must offer benefits to patients in the same groups as those recruited (e.g. to people with learning disabilities or children in general)

involvement of incompetent patients in research, the key points of which are listed in Box 3. The essence of these guidelines is that, unless incompetent patients participate in research, as a group either they will not benefit from it or benefit will be retarded as advances resulting from trials on competent patients will be applied on an individual and ad hoc basis. We believe that a similar public interest argument is valid in the use of case material without consent if it is not possible to gain consent, provided that the authors adhere to the criteria listed in Box 4 . This change of principle would primarily but not exclusively affect psychiatry, learning disability studies and paediatrics.

\section{Conclusions}

Some of the case studies used in psychiatry for teaching, learning and training are only useful because they include patients' personal (and therefore potentially identifying) information. In ideal practice, patients would give informed consent and the information would be anonymised as far as possible. Although many patients with psychiatric illness are capable of giving consent, there are those

Box 4 Criteria justifying the use in teaching or educational publications of case material without consent

- The cases should contribute significantly to learning, particularly that related to the patient group as a whole

- The information should be anonymised as far as possible

- No imaginary case could be substituted

- The authors should be able to prove that the patient was unlikely ever to be able to consent or to consent within the significant window of opportunity for learning from the case 
who are not capable and those who are capable but refuse. Furthermore, anonymisation is not always successful. Where no alternative - such as credible fictionalisation - is available, there is a limited public interest argument for using personal information without consent for teaching and learning purposes, even if this also involves publication, provided that as much identifying information as possible has been removed. The public interest defence for disclosure is always made on balance, with an assumption in favour of maintaining confidentiality.

Part of the harm caused to someone whose personal information is disclosed results from a sense of violation. We need to follow a principle of non-violation but it should perhaps be applied judiciously. It would not be in the public interest to apply this principle too rigorously, as this would prohibit the use of any personal information without consent, even that which was completely unidentifiable. This argument has already influenced limited disclosure for epidemiological research and should extend to information disclosed for teaching, even though the risks of violation in this case are greater and the material might be published. This justification applies particularly to material that requires urgent public and transparent discussion, or cases concerning those who are incapable of giving consent and are likely to remain so for so long as to render redundant eventual use with consent. Thus, an argument can be made for relaxing to this limited degree the demand of most medical journals that case material will not be published without consent.

\section{References}

BMJ Publishing Group (2004) BMJ Editorial Policies. http:// bmj.bmijournals.com/advice/editorial_policies.shtml.

Court, C. (1995) GMC finds doctors not guilty in consent case. BMJ, 311, 1245-1246.

General Medical Council (2004) Confidentiality: Protecting and Providing Information. http:/ /www.gmc-uk.org/standards/ secret.htm.

Rogers, W. \& Draper, H. (2003) The ethics of confidentiality in medical ethics. Journal of Medical Ethics, 29, 220-224.

Royal College of Psychiatrists (2004) Advances in Psychiatric Treatment: Instructions for Authors. http://apt.rcpsych.org/ misc/ifora.shtml.

Stone, D. (2001) Confidentiality, access to health records and the Human Rights Act 1988. In Healthcare Law: Impact of the Human Rights Act 1998 (eds A. Garwood-Gowers, J. Tingle \& T. Lewis), pp. 127-146. London: Cavendish Publishing.

\section{MCQs}

1 Which of the following best describes the authors' views of the advantages of using case studies in teaching and learning?

a case studies are a simple way of bringing together a set of complex issues related to the patient's circumstances, prognosis, diagnosis and treatment

b case studies enable potentially dry theoretical issues to be portrayed vividly, disseminate good practice, highlight new or emerging issues and help to challenge current practice

c case studies enable patients to learn by examples that are accessible to them, help medical students orient themselves to the medical setting, and reorient doctors who have had career breaks

d case studies can be disseminated widely and readily, for instance on the internet or in journals.

2 Rate the following as true or false:

a in English law, there is no proxy consent for incompetent adults

b doctors have a duty to act in the best interests of incompetent patients

c parents can give consent for minors

$\mathrm{d}$ doctors have a duty to consent on behalf of their incompetent patients.

3 The following reflect $A P T^{\prime}$ 's instructions to authors:

a fictional case studies are inferior to real case studies

b evidence of a patient's consent to use a case study is required

c patients should review material that is to be published

d when a patient is incompetent to consent, authorised consent can be substituted.

4 The following describe the conclusions of the authors:

a the public interest argument can, in certain circumstances, be extended to include the publication of case material without consent

b author affiliation can be an obstacle to anonymisation of case histories

c no distinction should be drawn between personal experience of violation and violation in principle

$\mathrm{d}$ that the patient recognises herself is not a sufficient criterion on which to base a claim that anonymisation has failed.

5 Protecting privacy as a matter of promise-keeping, regardless of the consequences, may best be described as an example of:

a virtue-based reasoning

b Kantian reasoning

c consequentialism

d appeal to human rights. 